\title{
Performance of update propagation techniques for data grid
}

\begin{abstract}
Data replication is a well known technique used to reduce accesses latency, improve availability, and performance in a distributed computing environment. An asynchronous replication is a commonly agreed solution for the consistency of replicas problem. Direct mail Update propagation is widely used in many replica management frameworks for replica consistency, however it suffers from high overhead at the master replica. we presents a new update propagation protocol called update propagation grid (UPG) to implement load balancing and more reliable system. We model the direct-mail scheme and UPG by a multiclass network queuing model and study its performance with different system load. The analytical solution shows that the UPG better performance than direct mail protocol.
\end{abstract}

